\title{
Efficacy of verum and sham acupoint catgut embedding for treatment of obesity: Study protocol for a randomized controlled trial
}

\author{
Yu-Mei Zhou ${ }^{\dagger} \mathbb{D}$, Bing Yan $^{\dagger}$, Wei-Qu Yuan ${ }^{\dagger}$, Hai-Bo Yu and Zhuo-Xin Yang ${ }^{*}$
}

\begin{abstract}
Background: Obesity has become a major public health hazard with epidemic proportions, affecting adults, adolescents, and children of both genders. Previous studies have suggested that acupoint catgut embedding (ACE) might be a potential therapeutic approach for obesity. The purpose of this study is to conduct a rigorous and normative trial to determine the efficacy of ACE for obesity.

Methods/design: A total of 99 eligible patients diagnosed with obesity will be recruited in this study. They will be randomly allocated to either the verum ACE group, sham ACE group, or waiting list (WL) group, with 33 patients in each group. Each patient in the two ACE-based groups will receive eight sessions of treatment, lasting over 8 weeks. The primary outcome is the reduction of body mass index (BMI) after treatment. Secondary outcomes will include waist circumference (WC), hip circumference (HC), waist:hip ratio, body fat percentage, blood lipid level, subcutaneous fat area, visceral fat area, and World Health Organization Quality of Life (WHOQOL). All the outcomes will be evaluated at baseline, at the end of the 8 weeks of treatments, and at 3 months of follow-up. The evaluators and data analyzers will be blinded to group allocation.
\end{abstract}

Discussion: The findings of this randomized, sham-, and WL-controlled trial will help to investigate the influence of ACE on clinical variables as well as visceral fat area of obesity, which will provide high-quality evidence on the efficacy of ACE for obesity.

Trial registration: Chinese Clinical Trial Registry, ChiCTR1800020248. Registered on December 21, 2018.

Keywords: Acupoint catgut embedding, Obesity, Randomized controlled trial, Visceral fat area

\section{Background}

Obesity is an increasing global public health issue characterized by the rise of body fat tissues. Genetic, dietary, lifestyle, and environment factors can all induce obesity. The prevalence of obesity has doubled over the past 10 years [1]. In 2015, in the US, more than $20 \%$ of the population was identified to be overweight or obese, being very common in adults [2]. According to Global Health Observatory (GHO) data released in 2016, 39\% of adults suffered from overweight or obesity. To date,

\footnotetext{
* Correspondence: 001188@gzucm.edu.cn

${ }^{\dagger}$ Yu-Mei Zhou, Bing Yan and Wei-Qu Yuan contributed equally to this work. The Fourth Clinical Medical College of Guangzhou University of Chinese Medicine, Shenzhen 518033, GuangDong, China
}

the prevalence of obesity is increasing in both developed and developing countries. Obesity is associated with increased risk for developing a range of comorbid conditions, such as type 2 diabetes (T2D) [3], cardiovascular disease (CVD) [4], fatty liver disease, and gastrointestinal and psychological disorders [5]. For instance, a number of scholars have reported that obese adults showed a $50 \%$ increased risk of developing T2D compared with normal-weight adults [6] and were twice as likely to have hypertension and CVD [7]. Nowadays, obesity and its complications impose a heavy burden on socioeconomic development; the World Health Organization (WHO) considers obesity as one of the most serious public health problems worldwide $[8,9]$. 
Methods to treat obesity such as lifestyle modification (specifically dietary modification and regular exercise), surgery, drugs, and complication therapy, are all referred to by the guidelines released by the National Heart, Lung and Blood Institute (NHLBI) of National Institutes of Health (NIH), American College of Cardiology (ACC), etc. [10]. The primary and the most valid recommendations for treating obesity are to restrict the intake of high calorie diets and more physical activities. However, these two methods are often challenging for patients to adopt long-term due to lifestyle and economic issues [11]. Additionally, according to the Cochrane Database of Systematic Reviews, there is no sufficient evidence to indicate that the short-term adjustment of food consumption and physical activity leads to long-term weight loss $[12,13]$. On the other hand, anti-obesity drugs seem to be questionable in terms of their efficacy and safety as the adverse events (AEs) associated with these drugs often have negative consequences, such as headache, dizziness, nausea and vomiting, insomnia, etc. The US Food and Drug Administration (FDA) in 2010 [14, 15] recommended against and prohibited the use of some antiobesity drugs because of serious liver damage and the high risk of CVD. Additionally, rimonabant was found to induce anxiety, depression, and other mental disorders [16]. Consequently, identification of effective and low-risk interventions is highly essential for obese individuals.

Acupoint catgut embedding (ACE), a complementary and alternative therapy, has been used for several decades to treat a variety of disorders, such as perimenopausal syndrome, chronic urticaria, depressive disorder, refractory insomnia, obesity, sciatica, etc. [17]. ACE involves the weekly infixing of surgical chromic catgut sutures into the subcutaneous tissue of the extremities and abdomen with a specialized needle under aseptic conditions. Owing to the continuous acupoint stimulation by the implanted sutures, ACE was considered to be more effective than ordinary acupuncture or electroacupuncture for patients with obesity [18]. Previous research revealed that the body weight and body mass index (BMI) of obese patients could be remarkably decreased after ACE therapy [19]. Moreover, ACE could adjust the imbalance of obesity-related hormones, e.g., leptin, ghrelin, and adiponectin, to reduce body weight $[19,20]$. In addition, visceral fat accumulation is often accompanied by obesity, which has been recently reported to play a vital role in the development of metabolic syndrome, a cluster of diabetes, dyslipidemia, and hypertension [21]. A randomized controlled trial (RCT) conducted by Lei et al. [22] revealed that electroacupuncture treatment could reduce BMI and waist circumference (WC), as well as visceral fat area (VFA). However, whether ACE can improve the VFA of obese patients and what the relationship is between VFA and obesity-related indices are still uncertain. Only a limited number of RCTs have investigated VFA changes with regard to ACE treatment for obesity.

Hence, this study is designed as a RCT to assess the effectiveness and safety of ACE for treatment of obesity.

\section{Methods/design}

\section{Study design}

This single-center, randomized, sham-controlled trial will be conducted at Shenzhen Traditional Chinese Medicine Hospital (Shenzhen, China). The study protocol was approved by the Ethics Committee of Shenzhen Traditional Chinese Medicine Hospital. The study will be conducted in accordance with the 1975 Declaration of Helsinki and the Standard Protocol Items: Recommendations for Interventional Trials 2013 Checklist (Additional file 1). A flowchart of the research procedure is shown in Fig. 1.

\section{Patient recruitment}

Patients who meet the inclusion criteria will be mainly recruited through outpatient clinics, online or offline advertisements (e.g., newspaper, poster, websites), or the WeChat public account of Shenzhen Traditional Chinese Medicine Hospital. If a patient is interested in joining the study, he/she can contact and consult with one of the researchers. Those patients who meet the inclusion criteria will be involved in the study. All the participants will sign a written informed consent form prior to the start of the study. The schedule of patient enrolment, intervention, and assessment is illustrated in Fig. 2.

\section{Inclusion criteria}

Inclusion criteria are as follows:

(1) Diagnosed with obesity according to Asian adult BMI criteria defined and proposed by the WHO Western Pacific region obesity working group in 2000

(2) WC of $\geq 90 \mathrm{~cm}$ for males or $\geq 80 \mathrm{~cm}$ for females

(3) Aged 18 to 65 years old

(4) Does not have any taboos regarding catgut embedding therapy

(5) Completes written informed consent

\section{Exclusion criteria}

Patients with any one of the following criteria are excluded from this trial:

(1) Endocrine disease (thyroid disease, pituitary disease, and diabetes mellitus) and autoimmune disease (systemic lupus erythematosus, Sjogren's syndrome, and rheumatoid arthritis)

(2) Metabolic disease such as hypertension and dyslipidemia 


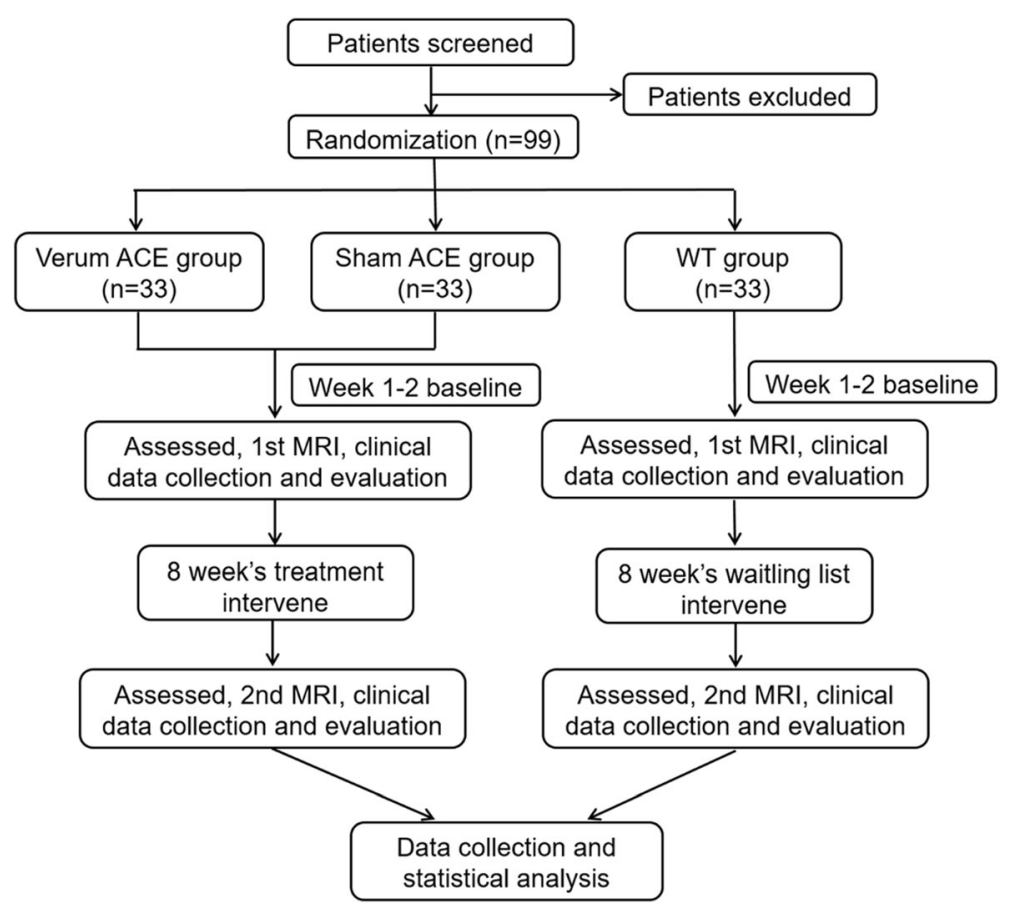

Fig. 1 Flowchart of the study design

(3) Other methods being used to control body mass and abdominal circumference, such as surgery, drugs, etc.

(4) Women in pregnancy, nursing, perimenopause

(5) Some severe diseases of the heart, liver, and kidney, or has tumor

(6) Diagnosed with a psychiatric disorder

(7) Participated in another study within 3 months

\section{Dropout criteria}

(1) Patients who do not meet the inclusion criteria but are mistakenly enrolled

(2) Occurrence of severe AEs or complications which result in stopping the trial

\section{Sample size}

The sample size has been calculated on the basis of results from a previous clinical trial [19]. The primary outcome in the present study is BMI reduction from baseline following 2 months of treatment. According to a previous study [19], in the ACE group, the BMI decreased 1.65 (standard deviation (SD) 1.24); in the sham ACE group, the BMI reduced 0.38 (SD 1.51). Considering a two-sided significance level of 0.05 and power of 0.90, 26 participants in each group are required as calculated by $t$ test in $G^{*}$ Power software (version 3, Institute for Experimental Psychology, HeinrichHeine-University, Germany). A dropout rate of $15 \%$ was taken into account so a total of 99 participants will be recruited in this trial ( $n=33$ participants in each group).

\section{Randomization and allocation concealment}

If the participants meet all the inclusion criteria and sign the written informed consent form, they accept the principle of random allocation. Hence, 99 eligible participants will be randomly assigned to the verum ACE group, sham ACE group, and waiting list (WL) group at a ratio of 1:1:1. A random allocation sequence number will be generated using Statistical Analysis System software (SAS, version 9.1.3, SAS Institute Inc., Cary, NC, USA) by an independent statistician who will not be involved in the treatment or data collection. The sequential numbers are written on cards and sealed in an opaque envelope by an independent research assistant. After a participant randomly selects an opaque envelope and obtains an allocation sequence number, a research assistant will assign an identification code to them and record them in a case report form (CRF). Then, the result of a patient's allocation will be given to the acupuncturists.

\section{Blinding}

The acupuncturists are not blinded for the entire process. To eliminate potential bias, outcome assessors and statisticians are blinded to group assignment. Patient allocation will only be revealed under some special conditions, such as severe allergy, serious infection, uncontrolled pain, etc. 


\begin{tabular}{|c|c|c|c|c|c|}
\hline \multirow[b]{3}{*}{ TIMEPOINT } & \multicolumn{5}{|c|}{ STUDY PERIOD } \\
\hline & \multicolumn{2}{|c|}{ Baseline } & \multicolumn{2}{|c|}{ Treatment phase } & \multirow{2}{*}{$\begin{array}{l}\text { Follow-up } \\
\text { Follow-up } \\
\text { (22th week) }\end{array}$} \\
\hline & 0 week & 1-2th week & $\begin{array}{l}\text { 2-10th week of } \\
\text { treatment }\end{array}$ & $\begin{array}{l}\text { Post of treatmet } \\
\text { (10th week) }\end{array}$ & \\
\hline \multicolumn{6}{|l|}{ ENROLMENT: } \\
\hline Eligibility screen & $x$ & & & & \\
\hline Informed consent & $\times$ & & & & \\
\hline Randomization & & $x$ & & & \\
\hline \multicolumn{6}{|l|}{ INTERVENTIONS: } \\
\hline Verum ACE group $(n=33)$ & & & $x$ & $x$ & $x$ \\
\hline Sham ACE group $(n=33)$ & & & $x$ & $\times$ & $x$ \\
\hline Waiting-list $(n=33)$ & & & $\bullet$ & $\longrightarrow$ & $x$ \\
\hline \multicolumn{6}{|l|}{ MRI SCAN: } \\
\hline Verum ACE group $(n=33)$ & & $x$ & & $x$ & $x$ \\
\hline ShamACE group $(n=33)$ & & $x$ & & $x$ & $x$ \\
\hline Waiting-list $(n=33)$ & & $x$ & & $x$ & $x$ \\
\hline \multicolumn{6}{|l|}{ ASSESSMENTS: } \\
\hline BMI & & $x$ & & $x$ & $x$ \\
\hline WC, HC, WHR, BFP & & $\times$ & & $x$ & $\times$ \\
\hline basal metabolic rate, $\mathrm{BP}, \mathrm{HR}$ & & $x$ & & $\times$ & $x$ \\
\hline $\mathrm{TC}, \mathrm{TG}, \mathrm{HDL}$ & & $x$ & & $x$ & $x$ \\
\hline WHOQOL & & $\times$ & & $\times$ & $x$ \\
\hline \multicolumn{6}{|l|}{ PARTICIPANTS SAFETY: } \\
\hline Adverse events & & & $x$ & $x$ & $x$ \\
\hline
\end{tabular}

Fig. 2 Study schedule for data collection

\section{Interventions}

The ACE intervention is compliant with the Standards for Reporting Interventions in Clinical Trials of Acupuncture (STRICTA) guidelines. Patients in the verum ACE group, sham ACE group, and WL group will receive real ACE therapy, sham ACE therapy, and delayed active ACE therapy 20 weeks later, respectively. The course for the two ACE groups will comprise eight sessions over 8 weeks (one session per week).

\section{Verum ACE group \\ Acupoints}

ACE treatment is a semi-standard method in this study. According to clinical practice, the literature, and the basic theory of traditional Chinese medicine, the prescription of ACE includes six obligatory acupoints and two groups of adjunct points.

The obligatory acupoints will include RN 12 (Zhongwan), ST 25 (Tianshu), SP 15 (Daheng), RN 06 (Qihai),
RN 04 (Guanyuan), and GB 26 (Daimai). Adjunct acupoints will consist of two groups: acupoints of group 1 involve ST 36 (Zusanli), SP 09 (Yinlingquan), and ST 40 (Fenglong); acupoints of group 2 include BL13 (Feishu), BL20 (Pishu), and BL23 (Shenshu). The obligatory acupoints will be chosen in each session, and the adjunct points will be selected every other session. All acupoints will be localized according to the names and locations of acupoints drafted in 2006 by the National Standard of the People's Republic of China (GB/T 12346-2006). Locations of the acupoints are presented in Table 1 and Fig. 3.

\section{Preparation of ACE}

Catgut (Suzhou medical Co. Ltd, Jiangsu, China) of length $1.5 \mathrm{~cm}$ will be placed in front of an embedded needle (Zhengjianggaoguan medicine Co., Ltd., Shenzhen, China) for the operation. ACE will be performed under sterile conditions. 
Table 1 Locations of acupoints in the two ACE groups

\begin{tabular}{|c|c|}
\hline Acupoints & Locations \\
\hline RN 12 (Zhongwan) & On the anterior median line, 4 cun above the umbilicus \\
\hline ST 25 (Tianshu) & Level with the umbilicus, and 2 cun lateral to the anterior median line \\
\hline SP 15 (Daheng) & Level with the umbilicus, and 4 cun lateral to the anterior median line \\
\hline RN 06 (Qihai) & On the anterior median line, 1.5 cun below the umbilicus \\
\hline RN 04 (Guanyuan) & On the anterior median line, 3 cun below the umbilicus \\
\hline GB 26 (Daimai) & $\begin{array}{l}\text { On the lateral abdomen, at the intersection of the vertical line of the free } \\
\text { end of the } 11 \text { th rib and the horizontal line on the same level of umbilicus, } \\
\text { or } 1.8 \text { inch below LR } 13 \text { (Zhangmen) at the liver meridian }\end{array}$ \\
\hline ST 36 (Zusanli) & $\begin{array}{l}3 \text { cun directly below ST } 35 \text { (Dubi), and } 1 \text { digit lateral to the anterior margin } \\
\text { of the tibia }\end{array}$ \\
\hline SP 09 (Yinlingquan) & $\begin{array}{l}\text { On the medial side of the calf, in the sunken spot between the medial lower } \\
\text { margin of the tibia and the medial margin of the tibia }\end{array}$ \\
\hline ST 40 (Fenglong) & $\begin{array}{l}8 \text { cun directly below ST } 35 \text { (Dubi), and } 2 \text { digits lateral to the anterior margin } \\
\text { of the tibia }\end{array}$ \\
\hline BL13 (Feishu) & $\begin{array}{l}\text { On the back, on the same level of the third subspinous of thoracic spine, and } \\
1.5 \text { cun lateral to the posterior midline }\end{array}$ \\
\hline BL20 (Pishu) & $\begin{array}{l}\text { On the back, on the same level of the } 11 \text { th subspinous of thoracic spine, and } \\
1.5 \text { cun lateral to the posterior midline }\end{array}$ \\
\hline BL23 (Shenshu) & $\begin{array}{l}\text { On the back, on the same level of the second subspinous of lumbar vertebra, } \\
\text { and } 1.5 \text { cun lateral to the posterior midline }\end{array}$ \\
\hline
\end{tabular}

\section{Operation procedures of ACE}

Firstly, the acupuncturist should disinfect their hands with $75 \%$ alcohol and don medical gloves, medical mask, and medical hat. Simultaneously, patients will lie on the bed, supine or in a prone position according to the location of the acupoints, and will fully expose the skin of the acupoint area. Secondly, the acupoint regions will be sterilized with iodophor and alcohol twice by the acupuncturist. Simultaneously, an assistant will place the catgut in front of an embedded needle. Thirdly, the acupuncturist will locate the acupoint and then embed the needle into the skin at the appropriate place; consequently, the catgut will be embedded into the acupoint at a depth of about $1.5,1$, or $1 \mathrm{~cm}$ at the abdomen, lower limbs, and back, respectively. The embedded needle will be withdrawn once the patient has a feeling of soreness
(Deqi); the catgut will be left under the tissue. Following this, sterile cotton balls will be pressed onto the acupoint for hemostasis and band-aids will be applied to the acupoints to prevent wound infection. Patients will be warned to avoid getting water on the embedded acupoints for $24 \mathrm{~h}$.

The frequency of the ACE treatment will be once per week, lasting a total of 8 weeks.

\section{Sham ACE group}

Patients in the sham ACE group will undergo a similar procedure to the verum ACE group except that nothing will be put into the catgut embedding needles before operation; thus, no catgut will be left under the patients' acupoint tissue after needle extraction. The prescription of acupuncture will also be the same as that in the verum ACE group.

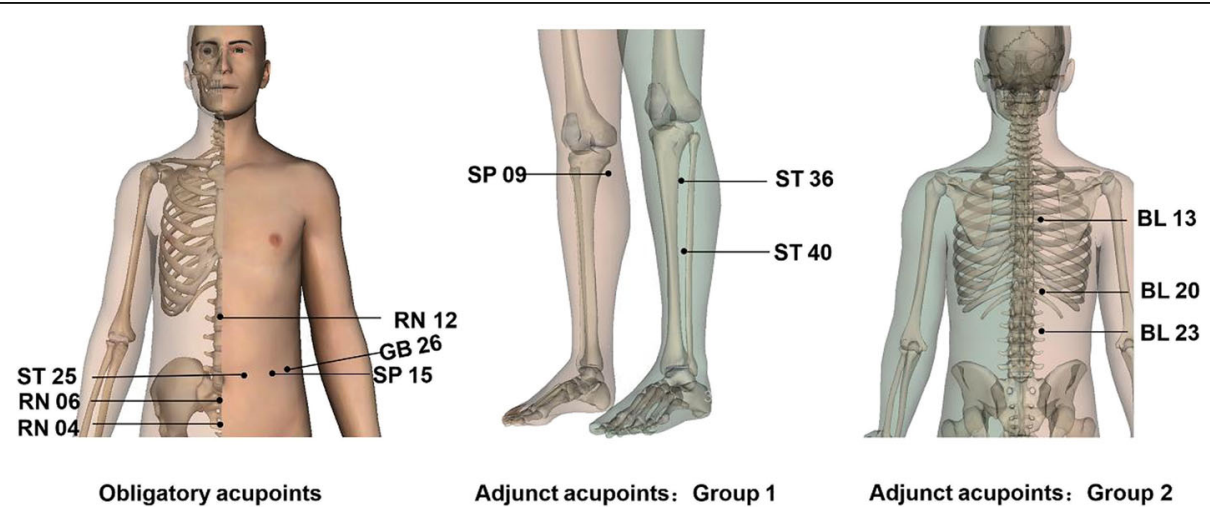

Fig. 3 Locations of acupoints 
In the current study, all patients will be treated separately to avoid influencing each other. Both groups will be treated under the same conditions by acupuncturists, who will receive special training before participation in the study. During the entire ACE operation process, the acupuncturists and assistants will not talk about details.

\section{WL group}

The WLC group will not have any intervention. The patients will be asked to receive delayed ACE therapy for free after a waiting period of 20 weeks.

\section{Magnetic resonance imaging data acquisition}

In this study, magnetic resonance imaging (MRI) images of subcutaneous adipose tissue and visceral adipose tissue will be acquired by a MRI scanner (MAGNETOM; Siemens, Munich, Germany) with a matrix body coil of 18 channels at the MRI Center of Shenzhen Traditional Chinese Medicine Hospital. Before scanning, the participants will be trained in deep-breathing exercises. During the scanning process, they will be asked to hold their breath for about $15 \mathrm{~s}$. The sequence parameters are as follows: flip angle, $65^{\circ}$; repetition time (TR)/echo time (TE), 195/3.69 ms; number of excitation (NEX), 1; matrix, $256 \times 131$; slice thickness, $7 \mathrm{~mm}$; and echo train length, 4 . In order to guarantee the passage of the image plane through the center of the vertebral disc between L4 and L5, the MRI data will be acquired from a sagittal scout. Qualitative image analysis will be performed by two independent reviewers as well.

\section{Outcome measurement}

The clinical outcomes will be used to assess patients' obesity levels and their quality of life. All measurements will be undertaken at baseline (after 8 weeks of treatments, and at 12 weeks of follow-up).

The primary outcome is the change of BMI from baseline. The BMI is calculated as follows: $B M I=$ mass $(\mathrm{kg}) /$ (height $(\mathrm{m}))^{2}$. According to the BMI-based criteria presented by the WHO and Asia-Pacific classifications in 2000, BMI scores are ranked as "23.0-24.9 (pre-obesity), " "25-29.9 (level I obesity)", and "more than 30 (level II obesity)".

The secondary outcomes will include WC, hip circumference (HC), waist:hip ratio (WHR), body fat percentage (BFP), and the WHO Quality of Life (WHOQOL). WC will be measured by a stretch-resistant tape at the midpoint between the top of the iliac crest and the lower margin of the least palpable rib. In addition, $\mathrm{HC}$ will be measured around the widest portion of the buttocks using a tape parallel to the floor [23]. BFP will be measured with bioelectrical impedance.

WHOQOL is a widely used questionnaire for measuring physical and mental health status. The WHOQOL scale includes 26 items, involving four domains of patient quality of life: physical, psychological, social, and environmental $[24,25]$. The total score ranges from 0 to 100. The lower the score, the poorer the patient's quality of life.

Other outcome parameters, including basal metabolic rate, blood pressure (BP), heart rate (HR), total cholesterol (TC), triglyceride (TG), and high-density lipoprotein (HDL) levels, will be tested at each time-point.

\section{Statistical analysis}

The statistical analysis will be conducted by independent statisticians who will be blinded to group allocation and intervention methods. Before data analysis, the research group will draw up a statistical plan, including the required data and method of data processing.

Data will be analyzed using SPSS 22.0 software (IBM, Armonk, NY, USA). For MRI data, the images will be analyzed on a workstation (Syngo Multimodality Workplace) for the quantification of VAT and SAT.

Demographic information and levels of measured variables will be analyzed by descriptive statistics. Categorical data are described as percentage $(\mathrm{n} \%)$ or analyzed using the Chi-square $\left(\mathrm{x}^{2}\right)$ test. Additionally, for continuous variables, if data are normally distributed, one-way analysis of variance (ANOVA) will be used to detect differences among the three groups. Otherwise, the Kruskal-Wallis $(\mathrm{K}-\mathrm{W})$ test can be considered. The longitudinal and repeated measured data will be analyzed by the repeated measure analysis.

In this study, SFA is quantified as an area between the outline of the abdominal skin and the outer abdominal muscle, while VFA is defined as the enterocoelia and retroperitoneal region between the inside edge of the abdominal muscles and the spinal front. Detailed methods have been described previously [26-28]. The correlation coefficients between two reviewers who analyzed the same image for SFA and VFA $(n=30)$ were $r=0.99, P<$ 0.001 and $\mathrm{r}=0.98, \mathrm{P}<0.001$, respectively.

Eventually, the Pearson's correlation between the changes of SFA and VFA and improvement of clinical variables will be calculated in each group.

For the above-mentioned statistical analyses, a $P$ value $<0.05$ is considered statistically significant.

\section{Safety}

To guarantee that the ACE operation is standard and safe, the acupuncturists in this study should pay attention to the following: (1) ensure the operation is strictly aseptic to prevent infection; (2) catgut should not be embedded in adipose tissue that could prevent fat liquefaction; (3) catgut should not be exposed to the body surface to prevent infection; (4) master the angle and depth of the embedding in order to avoid injury to the 
internal organs, great vessels, nerves, etc.; (5) acupoints can't be wet for $24 \mathrm{~h}$ after embedding; (6) inform patients that they may experience soreness, distension, and numbness at the acupoints for one or two days, and possibly more than three to five days.

\section{Management of adverse events}

ACE therapy may lead to different AEs, such as fainting during operation, subcutaneous hematoma, allergy, infection, severe pain, etc. Any AEs experienced by participants should be reported to the researchers. After confirming the validity of the AEs by an evaluator, the acupuncturists will immediately stop the treatment procedure and deal with the AEs. All AEs, as well as management of the AEs, will be carefully recorded during treatment and follow-up phases.

\section{Quality control}

Acupuncturists, assistants, data collectors, and statisticians who participated in the study should abide by the rules and regulations. Before the study, each researcher took basic study training to understand the design, purpose, and basic information about this research. The acupuncturists should have at least 3 years of practical acupuncture experience, and they also should be familiar with the operation process and be able to cope with any possible AEs. Data collectors are responsible for saving and managing various data, and strictly proofread data. Patient withdrawals and AEs during the study will be recorded in detail. Statisticians will be fully responsible for data management and statistical analysis. Regular team meetings will be held and fully documented.

\section{Ethics approval}

This study was approved by the Chinese Clinical Trial Registry. The registration number of this trial is ChiCTR1800020248. In addition, the study protocol was approved by the Ethics Committee of Shenzhen Traditional Chinese Medicine Hospital. Prior to start of the study, patients will be informed about the potential risks of the study. Patients voluntarily participate in the study with informed consent. If the protocol needs to be amended, all the materials on trial will be reported to the Ethics Committee, and the amended protocol can only be implemented after consent is acquired.

\section{Data management and monitoring}

The research associates will record the information on the CRFs and verify that the data is fully, swiftly, and accurately collected. The private information and medical records of patients, including their name, phone number, and ID number, will be anonymous to ensure confidentiality. All the research documents will be kept in specialized cabinets and preserved for at least 5 years after publication.

In addition, a data monitoring committee is established consisting of experienced experts in the Good Clinical Practice Department of the Shenzhen Traditional Chinese Medicine Hospital to periodically review the progress of the trial and monitor collection of the data, allocation concealment, etc. The modification or termination of the trial can be performed by the committee. The data monitoring committee is independent from the sponsor and has no conflict of interest.

\section{Discussion}

It is noteworthy that ACE is extensively applied for weight loss. Although previous studies have shown that ACE might be effective in improving overweight, highquality trials with rigorous design are still urgently required to assess the effects of ACE on obesity [29]. The results of the present study may contribute to a better understanding of how ACE exerts its potential therapeutic effects on obesity.

Recently, increasing attention has been paid to the visceral fat of obesity, which is related to energy storage, and is considered as an endocrine and paracrine organ, influencing a number of metabolic processes by releasing cytokines and bioactive mediators [30-33]. Body weight can be adjusted by cytokines and mediators. Thus, VFA is a significant indicator for estimating metabolic risk in obese populations $[34,35]$. In previous studies, however, the influence of ACE on obesity was mainly assessed by BMI or WC, and a limited number of studies have assessed those influences in relation to VFA. Hence, evaluation in this study is done according to both standard obesity-related indices (BMI, WC, etc.) and VFA in order to clearly illuminate the effects of ACE on obesity.

The purpose of this study is to compare measurement changes after 2 months of treatment with three different intervention methods: verum ACE, sham ACE, and no intervention (WLC group). Several studies [36, 37] have indicated that a sham-controlled design could separate specific and non-specific effects, which may play a pivotal role in evaluating the effectiveness of treatment. Thus, the ACE and sham ACE treatments are both applied to investigate the anti-obesity effects in this study. The sham ACE treatment involves only needle piercing into the acupoints without catgut being fixed; the rest of the procedure is the same as in the ACE group. Thus, it can successfully blind patients and estimators; as a result, it can minimize the placebo effect. In addition, as far as non-specific effects (sham ACE versus no intervention) and bias caused by psychological influences, the third group without any intervention is considered to avoid the placebo effects of group allocation or patients' beliefs on weight loss. A possible limitation of this trial 
is that compliance might be difficult for participants due to the long interval from the end of the 2 months of treatment to the follow-up at 3 months. Several actions, such as phone interview, should be taken to improve compliance.

The design and methodological rigor of this trial may hopefully provide consolidated evidence regarding the efficacy and safety of ACE for treating obesity, through collecting valuable and high-quality data, and also contribute to future research in ACE therapy.

\section{Trial status}

This trial is currently recruiting patients. The trial began recruitment on January 1, 2019 and is anticipated to be completed on December 31, 2021. The version number and date of the protocol are v1.0, and October 25, 2018, respectively.

\section{Supplementary information}

Supplementary information accompanies this paper at https://doi.org/10. 1186/s13063-019-3730-8

Additional file 1. SPIRIT 2013 checklist.

\section{Abbreviations}

ACC: American College of Cardiology; ACE: Acupoint catgut embedding; AE: Adverse event; BFP: Body fat percentage; BMI: Body mass index; BP: Blood pressure; CVD: Cardiovascular disease; FDA: Food and Drug Administration; GHO: Global Health Observatory; HC: Hip circumference; HDL: High-density lipoprotein; HR: Heart rate; MRI: Magnetic resonance imaging; NHLBI: National Heart, Lung and Blood Institute; NIH: National Institutes of Health; RCT: Randomized controlled trial; SD: Standard deviation; SFA: Subcutaneous fat area; STRICTA: Standards for Reporting Interventions in Clinical Trials of Acupuncture; T2D: Type 2 diabetes; TC: Total cholesterol; TG: Triglyceride; VFA: Visceral fat area; WC: Waist circumference; WHO: World Health Organization; WHOQOL: World Health Organization Quality of Life; WHR: Waist:hip ratio; WT: Waiting list

\section{Acknowledgements}

Thanks to all the patients in Shenzhen Traditional Chinese Medicine Hospital who provided valuable input to the trial.

\section{Authors' contributions}

YMZ, BY, and WOY contributed equally to this paper. ZXY is the corresponding author. YMZ participated in the conception and design of the trial. YMZ and WQY drafted the manuscript. HBY is in charge of the recruitment and treatment of patients. BY participated in data collection. WQY analyzed the data. ZXY proofread the final manuscript. All authors approved the submitted version of the manuscript.

\section{Funding}

This study is supported by the "Sanming Project" of Shenzhen Government (grant number SZSM201612001). The funder has no role in the design of the trial, collection, management, analysis, and interpretation of data, writing the manuscript, or submission of the manuscript for publication.

\section{Availability of data and materials}

The full data from this study will be available upon reasonable request after completion of the study.

\section{Ethics approval and consent to participate}

This study was approved by the Medical Ethics Committee of Shenzhen Traditional Chinese Medicine Hospital (Shenzhen Traditional Chinese Medicine Hospital Ethics approval (research) [2018] 77). All participants will sign written consent to participate in the study after being informed in detail about the study procedures.

\section{Consent for publication}

Not applicable. Results of the study will be published in papers in a peerreviewed academic journal or presented at relevant national and international conferences.

\section{Competing interests}

The authors declare that they have no competing interests.

Received: 8 April 2019 Accepted: 16 September 2019

Published online: 27 November 2019

\section{References}

1. Malik VS, Willett WC, Hu FB. Global obesity: trends, risk factors and policy implications. Nat Rev Endocrinol. 2013;9(1):13-27.

2. Yang L, Colditz GA. Prevalence of overweight and obesity in the United States, 2007-2012. JAMA Intern Med. 2015;175(8):1412-3.

3. Zhao L, Zhu L, Su Z, Li W, Li P, Liu Y, et al. The role of visceral adipose tissue on improvement in insulin sensitivity following Roux-en-Y gastric bypass: a study in Chinese diabetic patients with mild and central obesity. Gastroenterol Rep. 2018;6(4):298-303.

4. Narayan KM, Boyle JP, Thompson TJ, Gregg EW, Williamson DF. Effect of BMI on lifetime risk for diabetes in the U.S. Diabetes Care. 2007;30(6):1562-6.

5. Fruh SM. Obesity: Risk factors, complications, and strategies for sustainable long-term weight management. J Am Assoc Nurse Pract. 2017;29(S1):S3s14.

6. Bell JA, Kivimaki M, Hamer M. Metabolically healthy obesity and risk of incident type 2 diabetes: a meta-analysis of prospective cohort studies. Obes Rev. 2014;15(6):504-15.

7. Bastien M, Poirier P, Lemieux I, Despres JP. Overview of epidemiology and contribution of obesity to cardiovascular disease. Prog Cardiovasc Dis. 2014; 56(4):369-81.

8. Obesity: preventing and managing the global epidemic. Report of a WHO consultation. World Health Organ Tech Rep Ser. 2000;894:i-xii, 1-253.

9. Gregg EW, Jakicic JM, Blackburn G, Bloomquist P, Bray GA, Clark JM, et al. Association of the magnitude of weight loss and changes in physical fitness with long-term cardiovascular disease outcomes in overweight or obese people with type 2 diabetes: a post-hoc analysis of the Look AHEAD randomised clinical trial. Lancet Diabetes Endocrinol. 2016;4(11):913-21.

10. Jensen MD, Ryan DH, Apovian CM, Ard JD, Comuzzie AG, Donato KA, et al. 2013 AHA/ACC/TOS guideline for the management of overweight and obesity in adults: a report of the American College of Cardiology/American Heart Association Task Force on Practice Guidelines and The Obesity Society. J Am Coll Cardiol. 2014:63(25 Pt B):2985-3023.

11. Lang A, Froelicher ES. Management of overweight and obesity in adults: behavioral intervention for long-term weight loss and maintenance. Eur J Cardiovasc Nurs. 2006;5(2):102-14.

12. Mastellos N, Gunn LH, Felix LM, Car J, Majeed A. Transtheoretical model stages of change for dietary and physical exercise modification in weight loss management for overweight and obese adults. Cochrane Database Syst Rev. 2014;(2):Cd008066. https://doi.org/10.1002/14651858.CD008066.pub3.

13. Middleton KM, Patidar SM, Perri MG. The impact of extended care on the long-term maintenance of weight loss: a systematic review and metaanalysis. Obes Rev. 2012;13(6):509-17.

14. Food, Administration D. FDA drug safety communication: FDA recommends against the continued use of Meridia (sibutramine) M. 2014.

15. Food Administration D. FDA drug safety communication: completed safety review of Xenical/Alli (orlistat) and severe liver injury M. 2013.

16. Mitchell PB, Morris MJ. Depression and anxiety with rimonabant. Lancet (London, England). 2007;370(9600):1671-2

17. Steyer TE, Ables A. Complementary and alternative therapies for weight loss. Prim Care. 2009:36(2):395-406.

18. Huang CY, Choong MY, Li TS. Treatment of obesity by catgut embedding: an evidence-based systematic analysis. Acupunct Med. 2012;30(3):233-4.

19. Chen IJ, Yeh YH, Hsu CH. Therapeutic effect of acupoint catgut embedding in abdominally obese women: A randomized, double-blind, placebocontrolled study. J Women's Health (2002). 2018;27(6):782-90. 
20. Gucel F, Bahar B, Demirtas C, Mit S, Cevik C. Influence of acupuncture on leptin, ghrelin, insulin and cholecystokinin in obese women: a randomised, sham-controlled preliminary trial. Acupunct Med. 2012;30(3):203-7.

21. Matsuzawa $Y$. Therapy Insight: adipocytokines in metabolic syndrome and related cardiovascular disease. Nat Clin Pract Cardiovasc Med. 2006;3(1):35-42.

22. Lei H, Chen X, Liu S, Chen Z. Effect of electroacupuncture on visceral and hepatic fat in women with abdominal obesity: A randomized controlled study based on magnetic resonance imaging. J Altern Complement Med (New York, NY). 2017;23(4):285-94.

23. World Health Organization. Waist circumference and waist-hip ratio. Report of a WHO expert consultation. Geneva: WHO; 2008.

24. Development of the World Health Organization WHOQOL-BREF quality of life assessment. The WHOQOL Group. Psychol Med. 1998;28(3):551-8.

25. Skevington SM, Lotfy M, O'Connell KA. The World Health Organization's WHOQOL-BREF quality of life assessment: psychometric properties and results of the international field trial. A report from the WHOQOL group. Qual Life Res. 2004;13(2):299-310.

26. Tang L, Zhang F, Tong N. The association of visceral adipose tissue and subcutaneous adipose tissue with metabolic risk factors in a large population of Chinese adults. Clin Endocrinol. 2016;85(1):46-53.

27. Li X, Youngren JF, Hyun B, Sakkas GK, Mulligan K, Majumdar S, et al. Technical evaluation of in vivo abdominal fat and IMCL quantification using MRI and MRSI at 3 T. Magn Reson Imaging. 2008;26(2):188-97.

28. Thomas EL, Bell JD. Influence of undersampling on magnetic resonance imaging measurements of intra-abdominal adipose tissue. Int J Obes Relat Metab Disord. 2003;27(2):211-8.

29. Guo T, Ren Y, Kou J, Shi J, Tianxiao S, Liang F. Acupoint catgut embedding for obesity: Systematic review and meta-analysis. Evid Based Complement Alternat Med. 2015;2015:401914.

30. Janssen I, Katzmarzyk PT, Ross R. Waist circumference and not body mass index explains obesity-related health risk. Am J Clin Nutr. 2004;79(3):379-84.

31. Pischon T, Boeing H, Hoffmann K, Bergmann M, Schulze MB, Overvad K, et al. General and abdominal adiposity and risk of death in Europe. N Engl J Med. 2008;359(20):2105-20.

32. McGown C, Birerdinc A, Younossi ZM. Adipose tissue as an endocrine organ. Clin Liver Dis. 2014;18(1):41-58.

33. Oda E. The metabolic syndrome as a concept of adipose tissue disease. Hypertens Res. 2008;31(7):1283-91.

34. De Lorenzo A, Bianchi A, Maroni P, lannarelli A, Di Daniele N, lacopino L, et al. Adiposity rather than BMI determines metabolic risk. Int J Cardiol. 2013;166(1):111-7.

35. Shah NR, Braverman ER. Measuring adiposity in patients: the utility of body mass index (BMI), percent body fat, and leptin. PLoS One. 2012;7(4):e33308

36. Bacchetti $P$, Leung JM. Sample size calculations in clinical research. Anesthesiology. 2002;97(4):1028-9 author reply 1029-1032.

37. Irnich D, Salih N, Offenbacher M, Fleckenstein J. Is sham laser a valid control for acupuncture trials? Evid Based Complement Alternat Med. 2011;2011: 485945.

\section{Publisher's Note}

Springer Nature remains neutral with regard to jurisdictional claims in published maps and institutional affiliations.

Ready to submit your research? Choose BMC and benefit from:
- fast, convenient online submission
- thorough peer review by experienced researchers in your field
- rapid publication on acceptance
- support for research data, including large and complex data types
- gold Open Access which fosters wider collaboration and increased citations
- maximum visibility for your research: over 100M website views per year
At BMC, research is always in progress.
Learn more biomedcentral.com/submissions

